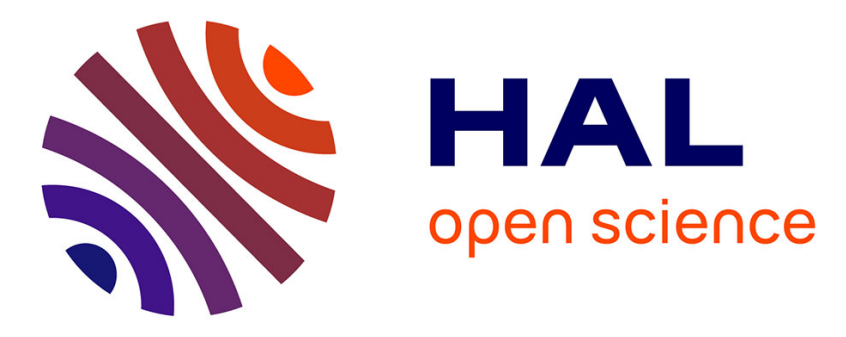

\title{
Design of a Low Complexity Interference Detector for LPWA Networks
}

Chhayarith Heng Uy, Carolynn Bernier, Sylvie Charbonnier

\section{To cite this version:}

Chhayarith Heng Uy, Carolynn Bernier, Sylvie Charbonnier. Design of a Low Complexity Interference Detector for LPWA Networks. I2MTC 2019 - IEEE International Instrumentation and Measurement Technology Conference, May 2019, Auckland, Nouvelle Zélande, New Zealand. 10.1109/I2MTC.2019.8827022 . cea-01972990

\section{HAL Id: cea-01972990 https://hal-cea.archives-ouvertes.fr/cea-01972990}

Submitted on 8 Jan 2019

HAL is a multi-disciplinary open access archive for the deposit and dissemination of scientific research documents, whether they are published or not. The documents may come from teaching and research institutions in France or abroad, or from public or private research centers.
L'archive ouverte pluridisciplinaire HAL, est destinée au dépôt et à la diffusion de documents scientifiques de niveau recherche, publiés ou non, émanant des établissements d'enseignement et de recherche français ou étrangers, des laboratoires publics ou privés. 


\section{Design of a Low Complexity Interference Detector for LPWA Networks}

\author{
Chhayarith Heng Uy \\ Univ. Grenoble Alpes \\ Gipsa-Lab, CEA, LETI \\ Grenoble, France \\ chhayarith.heng-uy@univ-grenoble-alpes.fr
}

\author{
Carolynn Bernier \\ Univ. Grenoble Alpes \\ CEA, LETI \\ Grenoble, France \\ carolynn.bernier@cea.fr
}

\author{
Sylvie Charbonnier \\ Univ. Grenoble Alpes \\ Gipsa-Lab, CNRS \\ Grenoble, France \\ sylvie.charbonnier@univ-grenoble-alpes.fr
}

\begin{abstract}
Many Internet of Things applications are deployed over shared ISM (Industrial, Scientific, Medical) radiofrequency spectrum bands. With the recent development of Low Power Wide Area (LPWA) wireless networks, the probability of interference and frame collisions has significantly increased. In this context, real-time interference monitoring is essential to provide precious information for network planning, base-station installation site selection, congested area detection, etc. This work presents a novel, low complexity interference detector for mobile LPWA nodes that is designed using a set of experimental data acquisitions. A first classifier is used to detect the presence of interference and shows a detection accuracy of $94 \%$. If interference is detected, a second classifier is used to classify the interference's relative strength into ten classes with a correct classification rate up to $97 \%$. The detector also provides an estimation of the interference's duration with an average relative error of $2 \%$ for medium to strong interference levels.

Index Terms-Internet of Things (IoT), Low Power Wide Area (LPWA), Interference Detection, Classification, Channel State Information, Channel Analysis Signal, Interference Presence Indicator, Ultra-Low Power Transceivers.
\end{abstract}

\section{INTRODUCTION}

The massive deployment of Internet of Things (IoT) applications requires an efficient use of the scarce radiofrequency (RF) spectrum. Current IoT wireless communication protocols are deployed either over cellular networks, using e.g. the NBIoT protocol, or in shared ISM (Industrial, Scientific, Medical) frequency bands. While cellular systems offer spectrum availability guarantees, spectrum sharing in ISM bands is only enforced through coexistence rules. Historically, ISM bands were used for short-range communication systems with inherently low mutual disturbance probability. The recent development of Low Power Wide Area (LPWA) wireless networks deployed over these same ISM bands has significantly increased the probability of interference for two reasons: First, the order of magnitude greater range increases the probability of co-located networks. This is true for both same-type and for differenttype co-located LPWA communication systems (e.g. networks such as LoRa, Sigfox, IEEE 802.15.4g/k, MYOTY, and so on, that are all deployed in the same ISM bands). Second, the increase in range implies that extremely low payload bitrates must be used. Thus, the resulting very long frame on-air times increase the probability of collisions [1]. For example, recent research has shown that LoRa networks based on an
ALOHA medium access protocol quickly become interference limited when node density increases, thus necessitating new interference-related performance metrics [2].

The present work focuses on the design of a low complexity, real-time interference detector for LPWA transceivers. Our objective is that, every time the transceiver correctly receives a frame, the detector simultaneously outputs information on the presence or absence of interference, and, if applicable, information concerning the interference's relative strength and duration. This interference-related information gathered by the wireless node can be used in many ways. An obvious application is network planning and selection of base station installation sites. Indeed, since the information is gathered by the nodes themselves, the network installer will dispose of very precise information concerning congested areas and be able to optimize the base station placement accordingly. Alternatively, interference-related information can be used by adaptive transmission protocols that aim to optimize the physical layer signalling rate in view of either improving throughput or saving energy when favourable RF propagation conditions have been detected. Detailed per-frame interference-related information can be exploited to fine-tune channel adaptation strategies.

This paper focuses on the design of an information-rich interference detector for ultra-low power transceivers typically employed in LPWA IoT applications. To comply with the extremely tight energy budget of these applications, our detection mechanism avoids the transmission of dedicated frames or symbols and avoids burdening the wireless node's processing capacity with algorithms that require high computational complexity. In addition, to ease the development of new MAC or network-level adaptive communication protocols, the information delivered by the detector is clear, simple to use, precise, and reflects the instantaneous propagation conditions.

To address this challenge, our strategy is to exploit channelrelated information contained within the received signal itself. To this end, a channel analysis signal (CAS) is defined in the digital base-band (DBB) processing part of the wireless receiver. The CAS is then processed to calculate two features per frame and which are used as inputs to classifiers. For each correctly received frame, the proposed detector is able to detect the presence or absence of interference, and if required 
provide an indication of the relative strength and duration of the interference. While the work described below is based on the LECIM FSK physical layer of the IEEE 802.15.42015 standard, the approach can easily be generalized to other LPWA communication standards.

The contributions of this paper are:

- A low complexity single-frame interference presence indicator (IPI) for LPWA transceivers based on a monofeature classifier and with $94 \%$ accuracy;

- A 10-level interference relative-strength classifier with an accuracy up to $97 \%$ built upon two minimal complexity features;

- An interference duration estimator;

- An experimentation-based classifier design methodology.

This paper is structured as follows: relative work is discussed in Section II, followed by a presentation of the experimental platform and data collection in Section III. The design methodology for our interference detector and interference strength estimator is proposed in Sections IV and V, respectively. Finally, experimental results are discussed in section VI.

\section{RELATED WORK}

Interference detection mechanisms have been developed and employed in the field of wireless communications for many different applications and in many differing contexts. In particular, blind interference detection techniques have been extensively investigated for cognitive radios (CR) where transceivers must compete for spectrum use while avoiding the interference caused by other communication systems sharing the same frequency band. For example, a pilot-aided interference detection method was proposed in [3] to allow robust orthogonal frequency division multiplexing (OFDM) signal detection in the presence of in-band interference. A pilotfree interference estimation technique was proposed in [4] for similar OFDM-based cognitive radios. More generally, many different spectrum sensing techniques have been developed in the cognitive radio context, for example using hidden Markov modeling [5].

Interference detection techniques have also been proposed for ISM radio communications where many different propa- gation schemes share the same spectrum. For example, for the $2.4 \mathrm{GHz}$ ISM band, an approach to detect and minimize the impact of interference caused by Bluetooth packets to IEEE 802.11g OFDM-based transmissions is proposed in [6]. The authors exploit the OFDM demodulation algorithm to extract useful information from the symbol magnitude for each subcarrier obtained after the demodulation FFT. In [7] and [8], the authors propose a PHY-independent interference detection mechanism based on the detection of abrupt changes in a BER estimate calculated for each received symbol. While very efficient, this approach requires that a large number of per-symbol confidence data, gathered within the $\mathrm{DBB}$, be transferred to the upper-layer protocol layers for processing. Alternatively, the authors in [9] propose a very simple interference detection mechanism based on the analysis of the distribution of coded bit errors within a frame. Unfortunately, this approach implies that frame data be necessarily encoded and limits interference detection to frames received at low SNR, since bit errors must absolutely be present.

Finally, in [10], demodulated chip error patterns of 2.4 $\mathrm{GHz}$ IEEE 802.15.4 transmissions are analyzed to infer the wireless link conditions, both to uncover the reasons for frame loss and also to determine whether interference is present, even when no frame is dropped. The extensive experiments performed by the authors focus on the transitional region of a wireless link which is characterized by highly volatile link conditions and thus in which received signal power is barely above the receiver's sensitivity. While a channel classification heuristic is proposed by the authors, little effort is committed to the explanation of observed error patterns with respect to the channel conditions. Compared to the above related work, our work focuses on LPWA transmission schemes which, with respect to OFDM signalling schemes, are generally of lower complexity. Consequently, we focus on low complexity interference detection mechanisms.

\section{EXPERIMENTAL PLATFORM AND DATA COLLECTION}

Since commercial off the shelf (COTS) devices do not allow access to DBB algorithms, a software defined radio is necessary for experimentation. The aim of the experiments is to gather realistic data for channel analysis, IPI choice, and

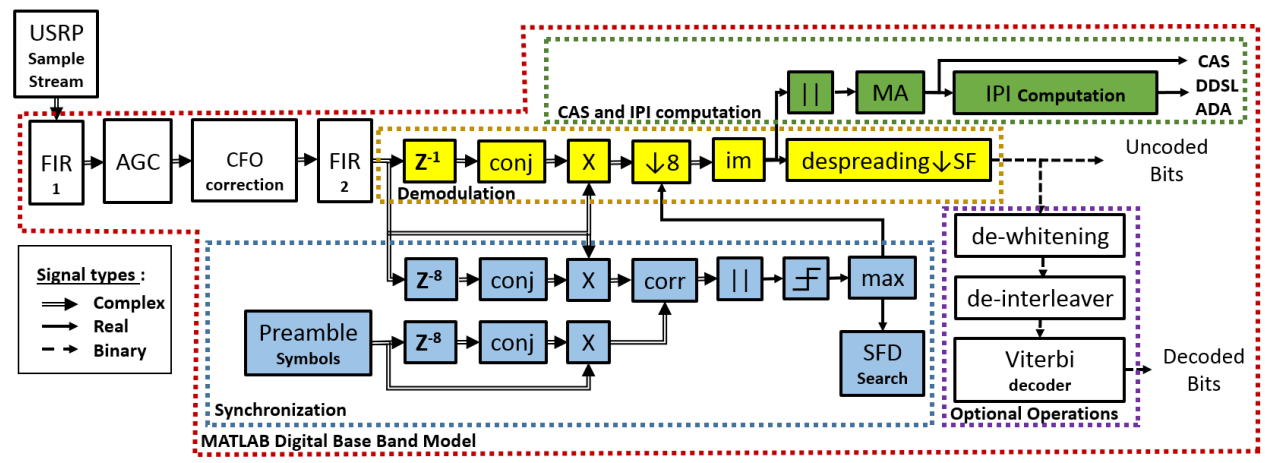

Fig. 1. IEEE 802.15.4-2015 LECIM FSK PHY digital baseband (DBB) receiver algorithm with CAS, DDSL and ADA computation. 
data-set collection for classifier training and validation. After the presentation of the SDR-based experimental platform, a brief description of the IEEE 802.15.4-2015 LECIM FSK PHY standard is given, and the labelled data-set collection procedure is described.

\section{A. SDR-Based Experimental Platform}

The SDR platform is composed of three universal software radio peripherals (USRP) located in a $5 \mathrm{~m} \times 4 \mathrm{~m}$ room. The three USRP are programmed to support the LECIM (Low Energy Critical Infrastructure Monitoring) FSK PHY of the IEEE 802.15.42015 standard [11]. The first USRP is used as transmitter $(T x)$, the second as interference source $(I x)$ and the last as receiver $(R x)$. The USRP are N210 models [12] from Ettus Research with WBX [40 MHz - $2200 \mathrm{MHz}$ ] daughter-board. All experiments employ a carrier within the 869.3-869.4 MHz ISM band which is not limited by duty cycle in France if transmissions are below $10 \mathrm{~mW}$. Attenuators are inserted between each emitting USRP output SMA port and the half-wave dipole antenna which allows us to limit the transmission range to approximately two meters which is necessary for leading controlled experiments. Each USRP is an RF front-end which performs up/down frequency conversion, analog filtering and DA/AD conversion. The USRPs are driven by GnuRadio through a Gbit Ethernet connection to a PC. The transmission and reception DBB algorithms are implemented in MATLAB which provides/receives complex baseband data at a sample rate of $300 \mathrm{kHz}$ to/from GnuRadio.

\section{B. IEEE 802.15.4-2015 LECIM FSK PHY}

Since FSK-based modulations are extremely common in ultra-low power communication systems, the experiments described in this work are based on the IEEE 802.15.4-2015 LECIM FSK PHY which extends the range of conventional IEEE 802.15.4 physical layers through spread spectrum techniques [13]. This physical layer employs the GMSK modulation at $37.5 \mathrm{kchip} / \mathrm{s}$ with optional direct-sequence modulation spreading factors of $2,4,8$ or 16 . The frames generated for the experiments are composed of a 4-byte preamble, 3byte start of frame delimiter (SFD), 2-byte header, 1250byte payload and 2-byte cyclic redundancy check (CRC) code. The 2-byte CRC is based on the generator polynomial $G(x)=x^{16}+x^{15}+x^{2}+1$. Convolutional $1 / 2$ rate forward error code with constraint length $K=7$, optional in the standard, is used in all of our experiments along with a spreading factor of 4. Including coding and frame synchronization overheads, the resulting frames have an on-air time of 2.15 seconds.

A digital baseband receiver able to detect FSK-modulated frames was designed and is presented in Fig. 1. Indeed, with $A[k]$ defined as the complex output of the channel filter (FIR2), it can be shown that $x[k]=\Im\left(A[k] \times A^{*}[k-1]\right)$ is a good approximation of the instantaneous frequency. Synchronization and SFD detection is handled by blocks in blue which perform a correlation with the known preamble sequence. These blocks calculate the optimal decimation moment thus allowing the sample stream to be decimated by a factor 8 . Demodulation is performed by yellow blocks while green blocks compute the channel analysis signal (CAS) as well as the features used by our interference detector (DDSL and ADA) which are described below.

\section{Labelled Data Collection}

The $T x$ power is chosen such that the received signal is approximately $15 \mathrm{~dB}$ above the $R x$ sensitivity. This relatively high SNR level is typical of real-world deployments since it allows for a comfortable fading margin. Labelled datasets are produced by two experiments. In the first one, 300 frames are acquired in an undisturbed RF environment and labelled accordingly. In the second experiment, an interference environment is generated using $I x$ as an interference source. The interference is an IEEE 802.15.4-2015 LECIM FSK PHY signal emitted in the same frequency band as that of $T x$. The emission duration of $I x$ is randomly selected in the range $[30 ; 1700] \mathrm{ms}$. To account for variable interference levels, ten interference environment data-sets are collected, each obtained using a different baseband signal amplitude ' $a$ ' applied to the samples transmitted by $I x$. Values of $a$ are selected from 0.1 to 1 with a step of 0.1 in order to cover a $20 \mathrm{~dB}$ dynamic range for received interference power. Thus, values of $a$ close to 0.1 and 1 respectively correspond to low and high levels of interference. The Ix USRP gain is configured such that, when $a=1$, frames received by $R x$ contain bit errors which are corrected by the forward error correction decoding process. In this configuration, the measured signal to cochannel interference ratio (CIR) is approximately $4 \mathrm{~dB}$, which is consistent with the expected sensitivity of an FSK-based spread-spectrum receiver. In all of these experiments, only CRC-valid frames were analyzed and collected for feeding the classifier training and evaluation steps. For each value of $a$, a set of 100 frames was acquired to which was assigned the label 'disturbed' as well as the label ' $l$ ', with $l=10 \times a$. Thus a total of 1000 frames was collected in this second experiment.

\section{Interference Detector Design Methodology}

In this section, the proposed channel analysis signal (CAS) and interference presence indicator (IPI) are presented. Then, the methodology for designing the interference detector and interference duration estimator is detailed.

\section{A. Channel Analysis Signal (CAS)}

In [7], [10] and [8], the receiver's digital baseband (DBB) is instrumented to compute channel analysis signals (CAS) for channel diagnostics. Similarly, the CAS proposed in this work is extracted directly from the receiver's DBB. In our case, the CAS corresponds to the (averaged) absolute value of the input signal of the soft de-spreading block, $x[k]$. The CAS is thus proportional to the probability that the received symbol is correct. More formally, the CAS is defined as:

$$
C A S_{k}=\frac{1}{\text { win }} \sum_{j=0}^{\text {win-1 }}|x[k-j]| .
$$




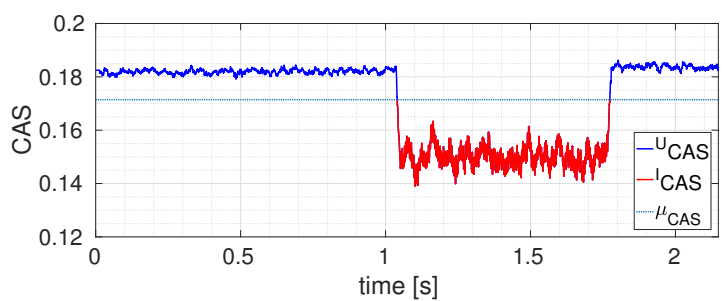

Fig. 2. Channel Analysis Signal (CAS) with an 'undisturbed' part $\left({ }^{U} C A S\right)$ in blue and an 'interference' part $\left({ }^{I} C A S\right)$ in red. $\mu_{C A S}$ is the mean value of the entire CAS.

In order to smooth the signal $|x[k]|$ and to highlight the interference footprint, a moving average (MA) over a computation window of win samples is used. The choice of win must be compatible with the shortest detectable interference duration. For the given $37.5 \mathrm{kHz}$ decimated sample rate, a value of win $=1000$, corresponding to $T_{w i n}=26.6 \mathrm{~ms}$, is selected. Finally, we define $C A S=\left[C A S_{1}, \ldots, C A S_{N}\right]$ as the channel analysis signal, where $N$ is the total number of samples in the frame. Fig. 2 shows an example of the CAS calculated for a frame received in the presence of interference. In this figure, we can easily recognize the temporal footprint of the disturbance corresponding to the presence of a strong interference. The computation of the CAS signal only requires a modulus operation and a moving average filter allowing this approach to be easily generalized to all FSK-based IoT standards (e.g. Sigfox, Bluetooth). Other signalling schemes can similarly be addressed by reusing the soft demodulator input signal of the corresponding DBB receiver.

\section{B. Interference Presence Indicator (IPI)}

Our aim is to find a single-value, low complexity IPI that is able to accurately extract the interference footprint information from the CAS. To this end, we propose to use the DDSL which measures the Difference between the averaged Disturbance and Signal Levels. The DDSL summarizes into one numerical value the strength footprint of the interference (disturbance caused by the emission of $I x$ used as interferer). Let $\mu_{C A S}$ be the mean value of the CAS over the frame. ${ }^{U} C A S_{k}$ is the set of CAS values such that $C A S_{k}>\mu_{C A S}$ and, correspondingly, ${ }^{I} C A S_{k}$ is the set of CAS values such that $C A S_{k} \leq \mu_{C A S}$ (cf. Fig. 2). DDSL is computed as (2) with $M$ and $P$ the number of samples respectively contained in the sets ${ }^{U} C A S_{k}$ and ${ }^{I} C A S_{k}$, with $M+P=N$.

$$
D D S L=\frac{1}{M} \sum_{k=1}^{M}{ }^{U} C A S_{k}-\frac{1}{P} \sum_{k=1}^{P}{ }^{I} C A S_{k}
$$

\section{Mono-Feature Classifier Based Detector}

The DDSL is used to classify each frame into two classes: 'undisturbed' and 'disturbed', with a detection threshold, $\lambda$. The 'disturbed' class is selected when DDSL is higher than $\lambda$. The value of $\lambda$ is learned using a training data set from an empirical ROC curve. The ROC curve displays the true positive rate as a function of the false positive rate for all possible values of $\lambda$. A true positive is defined as a frame with interference correctly classified as 'disturbed'. A false positive is defined as an undisturbed frame wrongly classified as 'disturbed'. The performance of an ideal detector is determined by a $100 \% \mathrm{TP}$ and a $0 \% \mathrm{FP}$, corresponding to the point $\{\operatorname{FPR}(\lambda), \operatorname{TPR}(\lambda)\}=\{0,1\}$. The optimum value of $\lambda$ is found by choosing the point on the ROC curve with the minimum distance from the ideal point $(0,1)$. The minimizing equation is described by (3).

$$
\lambda_{\text {opt }}=\underset{\lambda}{\operatorname{argmin}}\left\{\left[F P R(\lambda)^{2}+(1-T P R(\lambda))^{2}\right]^{1 / 2}\right\}
$$

\section{Interference Duration Estimator}

Once a frame is detected as 'disturbed', the duration of the interference can be calculated from the CAS signal. The estimated interference duration $\hat{\tau}$ is equal to the number of samples contained in the ${ }^{I} C A S_{k}$ set, $P$, divided by the decimated sample rate $\left(F_{s, d}\right) . \hat{\tau}$ is computed by (4).

$$
\hat{\tau}=\frac{P}{F_{s, d}}
$$

Alternatively, an estimation of the relative interference duration, defined as $P / N$, could also be calculated. Depending on how the interference detector data is exploited (e.g. network planning or adaptive protocol design), one or the other information might be more useful.

\section{INTERFERENCE StRENGTH ESTIMATOR}

In this section, each frame previously classified as 'disturbed' is further classified into one of ten classes, $C=$ $1,2, \ldots, 10$, corresponding to the previously defined labels $l=1,2, \ldots, 10$ related to the interference strength.

\section{A. Average of the Disturbance Area}

While only DDSL is needed for the interference detector, the 10-level classification requires the use of an additional feature, the average of the distance area (ADA), to perform at its best. ADA gathers additional details concerning the temporal footprint of the interference. ADA is defined as the area between the ${ }^{I} C A S_{k}$ and the $\mu_{C A S}$ curves (cf. Fig. 2) divided by the number of times the average, $\mu_{C A S}$, is intersected by the $C A S_{k}$ signal. The computation of ADA is described by (6). The calculation of ADA requires only a few mathematical operations since $\frac{1}{P} \sum_{k=1}^{P}{ }^{I} C A S_{k}$ and $\mu_{C A S}$ have been previously computed. Also, the comparisons used previously to separate the ${ }^{I} C A S_{k}$ and ${ }^{U} C A S_{k}$ sets can be simultaneously used to compute the denominator of (6).

$$
\begin{gathered}
S_{k}=\operatorname{sgn}\left(C A S_{k}-\mu_{C A S}\right) \\
A D A=\frac{2 P\left(\mu_{C A S}-\frac{1}{P} \sum_{k=1}^{P}{ }^{I} C A S_{k}\right)}{\sum_{k=2}^{N}\left|S_{k}-S_{k-1}\right|}
\end{gathered}
$$




\section{B. 10-Level Interference Classification}

The ten-level interference classifier uses ten multidimensional centroids. Each frame $i$ is represented by a set of 2 features $f_{i}=\left[D D S L_{i}, A D A_{i}\right]$. In the feature space, each class $C$ is represented by its centroid and dispersion. The centroid is defined as $\mu_{C}=\left[{ }^{D D S L} \mu_{C},{ }^{A D A} \mu_{C}\right]$ corresponding to the mean values of DDSL and ADA for each class. The dispersion of each class is represented by $V_{C}=\left[{ }^{D D S L} \sigma_{C}^{2},{ }^{A D A} \sigma_{C}^{2}\right]$, the variance values of DDSL and ADA. The values $\mu_{C}$ and $V_{C}$ are learned for each class using its corresponding learning data set. For a test frame $f_{i}$, the Mahalanobis squared distance between $f_{i}$ and the centroid of class C, $D_{i, C}$, is calculated for each class using (7). The covariance matrix of each class is considered to be diagonal.

$$
\begin{gathered}
D_{i, C}=\left(f_{i}-\mu_{C}\right)\left(\operatorname{diag}\left(V_{C}\right)\right)^{-1}\left(f_{i}-\mu_{C}\right)^{T} \\
\mathfrak{C}_{i}=\underset{C}{\operatorname{argmin}}\left\{D_{i, C}\right\}
\end{gathered}
$$

The class assigned to $f_{i}, \mathfrak{C}_{i}$ where $\mathfrak{C} \in \mathbb{N}^{[1,10]}$, is the class whose $D_{i, C}$ is the smallest of the ten computed. Note that, for each class, the computation of (7) requires only 2 subtractions, multiplications and divisions and one sum.

\section{Classifier Performance}

To evaluate the performance of this classifier, a correct classification rate can be computed for different requirement levels. The $\gamma$-correct classification rate, $R_{ \pm \gamma}$, is given by (9), counting as correct any frame classified into the $\gamma$ adjacent classes. In (9), $[\ldots]$ are the Iverson brackets, $[B]$ is defined to be 1 if $B$ is true, and 0 if it is false and $N_{T}$ is the number of frames contained within the test set.

$$
R_{ \pm \gamma}=\frac{1}{N_{T}} \sum_{i=1}^{N_{T}}\left[\left|\mathfrak{C}_{i}-l_{i}\right| \leq \gamma\right]
$$

\section{RESUlts}

\section{A. Disturbed versus Undisturbed Classifier}

A learning set made of 150 'undisturbed' frames and 150 'disturbed' (i.e., 'interference') frames randomly chosen among the 1000 samples labelled as 'interference' (cf. section III-C) is created. This set is used to build the ROC curve displayed in Fig. 3. Using (3), the optimum operating point (FPR=0.04, TPR $=0.82$ ) is found with $\lambda_{o p t}=3.4 \times 10^{-3}$. Fig. 4 shows clearly that it is possible to use DDSL to discriminate 'disturbed' frames from 'undisturbed' ones, with $\lambda_{o p t}$ as a good boundary. The presence of some error points in the DDSL distribution is explained by the fact that the ROC curve never reaches the ideal point $(0,1)$ meaning that the classifier is not flawless. Next, classifier performance is evaluated from a test set consisting of 300 labelled frames (150 frames per class) different from those contained in the learning set. The detector satisfies a correct detection rate of $94 \%$.

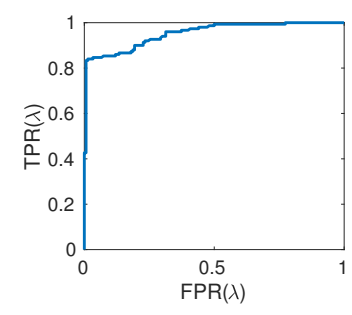

Fig. 3. ROC curve for classifying 'disturbed' versus 'undisturbed' frames with $\lambda$ as the varying threshold parameter using the DDSL feature.

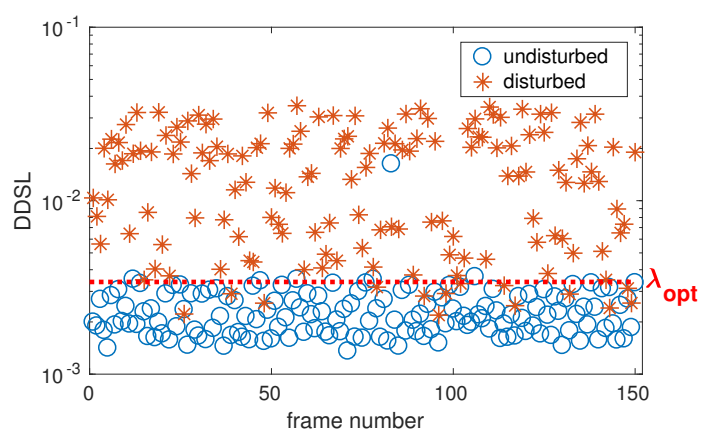

Fig. 4. Representation of the DDSL for the labelled input learning set with the optimum selected threshold $\lambda_{o p t}$.

\section{B. Interference Duration Estimator}

To validate and compute the interference duration estimator performance, an additional 400 frames are collected, this time with controlled interference duration. In order to generate a correct validation data-set, frames transmitted by $I x$ are set to have on-air times of $0.4 \mathrm{~s}$ separated by $0.4 \mathrm{~s}$ pauses while $T x$ transmits 2 second frames separated by 2 second pauses. In this way, we are certain that each acquired frame is impacted by at least 0.8 seconds of interference. The extraction of the ${ }^{I} C A S_{k}$ set is slightly modified to include only samples corresponding to a complete interference signal. To emulate various levels of interference, randomly generated values of $a$ between 0.2 and 1 are applied to $I x$. The interference duration estimator accuracy is evaluated computing the root mean square error (RMSE) and the average relative error $\left(\delta \alpha_{r}\right)$ between the estimated duration value $\hat{\tau}$ and the preset value $\tau$. Fig. 5 shows the evolution of the RMSE and the average relative error $\delta \alpha_{r}$ for different values of the interference's complex signal amplitude $a$ (cf. section III-C). The proposed interference duration estimator is very accurate for $a>0.5$, corresponding to medium to strong interference with a RMSE $<0.044 \mathrm{~s}$ and a $\delta \alpha_{r}<2 \%$. Even if the precision of the estimator is less accurate for lower-level interference, the estimator can still provide valuable information that can be used by an adaptive protocol that attempts to improve communication link by modifying frame length.

\section{Interference Strength Classifier}

The interference strength classifier is trained using a training set of 500 samples (50 samples per class). Next, the classifier 


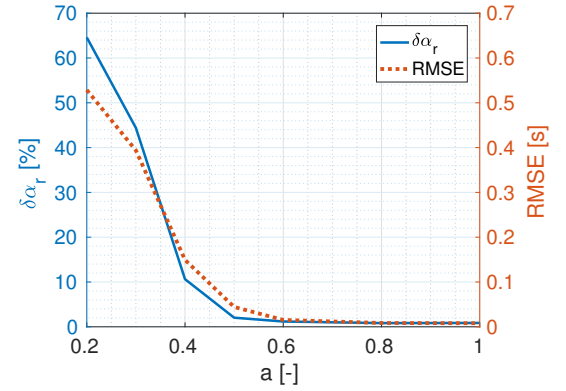

Fig. 5. Evolution of the average relative error $\delta \alpha_{r}$ and the RMSE of the duration estimation as a function of the interference's complex signal amplitude ' $a$ '.

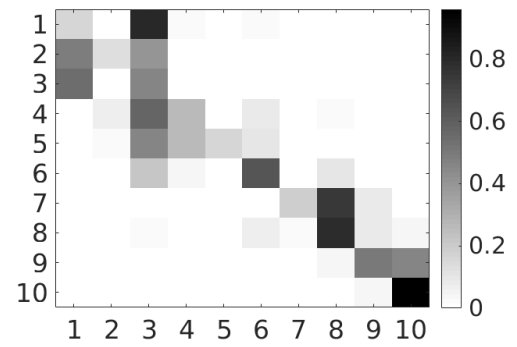

Fig. 6. Confusion matrix represented in gray scale computed from the test set classification.

performance is evaluated using a validation set consisting of the remaining 500 samples. The performance is summarized by the confusion matrix displayed in Fig. 6. Each row of the matrix shows the classifier output (in percentage) for the labelled set identified on the vertical scale. Thus, the gray-scale number located at $(\mathrm{i}, \mathrm{j})$ is the percentage of samples classified as belonging to class $\mathrm{i}$ while belonging to set $\mathrm{j}$. Correct classifications are presented in the diagonal while classification errors are represented by results outside of the diagonal. As expected, Fig. 6 shows a greater number of classification errors for classes 1, 2, 3 and 5 while, for the rest of the classes, the classification results are distributed in the diagonal area reflecting a good classification performance. Three $\gamma$-correct classification rates are computed: $R_{ \pm 0}=42 \%, R_{ \pm 1}=74 \%$ and $R_{ \pm 2}=97 \%$. This last value means that the classifier practically never assigns the frame into a class further than 2 classes away from the correct one. This shows its ability to accurately evaluate the level of the interference strength. Most importantly, the classifier never confuses a low interference frame with a strong interference frame.

\section{CONCLUSION}

In this paper, we present an information-rich interference detector able to detect the presence of a simultaneous transmission in a wireless channel while estimating its duration and relative strength. Since the detector provides this data for each correctly received frame, it is a valuable tool for monitoring the RF propagation conditions in real time. The low complexity detector is built upon a channel analysis signal (CAS) extracted directly from the receiver DBB, two novel feature extractors (DDSL and ADA) and two classifiers. Low computational complexity is an important requirement for all elements of the detector. The first classifier is able to detect the presence of interference with $94 \%$ accuracy. The second is able to discriminate between low, medium and strong interference levels with $97 \%$ accuracy. A reliable estimation of the interference duration, with an average $2 \%$ relative error when the level of interference is medium to strong is also proposed. The approach presented in this work can be easily generalized to other LPWAN standards. Future work will include experimentation with other types of interference sources (e.g. LoRa, Sigfox) to validate the classifier performance in generalized scenarios.

\section{ACKNOWLEDGMENT}

This work was supported by the LabEx PERSYVAL-Lab (ANR-11LABX-0025-01).

\section{REFERENCES}

[1] S. Rauh, J. Robert, M. Schadhauser, and A. Heuberger, "Lpwan occupancy model parameter identification for license exempt sub-ghz frequency bands," in 2018 11th German Microwave Conference (GeMiC), March 2018, pp. 111-114.

[2] O. Georgiou and U. Raza, "Low Power Wide Area Network Analysis: Can LoRa Scale?" IEEE Wireless Communications Letters, vol. 6, no. 2, pp. 162-165, Apr. 2017.

[3] T. Li, W. Mow, V. Lau, M. Siu, R. Cheng, and R. Murch, "Robust joint interference detection and decoding for OFDM-based cognitive radio systems with unknown interference," IEEE Journal on Selected Areas in Communications, vol. 25, no. 3, pp. 566-575, Apr. 2007.

[4] T. Zhao, H. Zhao, Y. Zhao, X. Cheng, and B. Ai, "A frequency-domain estimation scheme for single-tone interference in ofdm systems," in 2011 Third International Conference on Communications and Mobile Computing, April 2011, pp. 409-412.

[5] A. J. Coulson, "Spectrum sensing using hidden markov modeling," in 2009 IEEE International Conference on Communications, June 2009, pp. 1-6.

[6] S. Vogeler, L. Broetje, K. . Kammeyer, R. Rueckriem, and S. Fechtel, "Blind bluetooth interference detection and suppression for ofdm transmission in the ism band," in The Thrity-Seventh Asilomar Conference on Signals, Systems Computers, 2003, vol. 1, Nov 2003, pp. 703-707 Vol.1.

[7] K. Jamieson and H. Balakrishnan, "Ppr: Partial packet recovery for wireless networks," SIGCOMM Comput. Commun. Rev., vol. 37, no. 4, pp. 409-420, Aug. 2007.

[8] Mythili Vutukuru, Hari Balakrishnan, and Kyle Jamieson, "Cross-layer wireless bit rate adaptation," ACM SIGCOMM Computer Communication Review, vol. 39, no. 4, pp. 3-14, 2009.

[9] Filip Barac, Stefano Caiola, Mikael Gidlund, Emiliano Sisinni, and Tingting Zhang, "Channel Diagnostics for Wireless Sensor Networks in Harsh Industrial Environments," IEEE Sensors Journal, vol. 14, no. 11, pp. 3983-3995, Nov. 2014.

[10] Kaishun Wu, Haoyu Tan, Hoi-Lun Ngan, Yunhuai Liu, and Lionel M. $\mathrm{Ni}$, "Chip Error Pattern Analysis in IEEE 802.15.4," IEEE Transactions on Mobile Computing, vol. 11, no. 4, pp. 543-552, Apr. 2012.

[11] "IEEE Standard for Low Rate Wireless Networks-IEEE Std 802.15.42015."

[12] Ettus Research, "USRP Hardware Driver and USRP Manual-USRP2 and N2x0 Series." [Online]. Available: http://files.ettus.com/manual/ page_usrp2.html

[13] Y. Tada, Y. Sato, and S. Kato, "High deployability of ieee $802.15 .4 \mathrm{k}$ dsss systems in interference dominated bands," in Eigth International Conference on Sensor Technologies and Applications (SENSORCOMM), 2014. 